\title{
Adaptación y validación del MRBQ (Mathematics- Related Beliefs Questionnaire) al contexto colombiano con estudiantes de secundaria
}

\section{Adaptation and validation of the Mathematics-Related Beliefs Questionnaire (MRBQ) to the Colombian context with high school students}

\author{
Jose Manuel Diego-Mantecón ${ }^{1}$ \\ Francisco Javier Córdoba-Gómez²
}

\begin{abstract}
Resumen: Este artículo presenta la adaptación y validación del MRBQ (Mathematics-Related Beliefs Questionnaire) al contexto colombiano. El MRBQ es un instrumento, desarrollado en la Universidad de Lovaina (Bélgica), que evalúa las creencias de los estudiantes sobre las matemáticas y que ha sido validado en varios países. El instrumento fue administrado a 670 estudiantes de secundaria en centros públicos de la ciudad de Medellín (Colombia). Los análisis revelaron que las creencias sobre las matemáticas de estos estudiantes se representan mejor con un modelo de 10 factores, y no con uno de cuatro como sugieren estudios anteriores. Los 10 factores identificados explican más fielmente el modelo teórico original y muestran una varianza de 56.34\%, mayor que la obtenida en investigaciones previas. Estos resultados no solo sugieren mejoras significativas del MRBQ, sino que proporcionan la base para el desarrollo de una herramienta fiable que evalúe las creencias en el contexto colombiano. Hasta lo que estos autores conocen no se dispone de otro instrumento
\end{abstract}

Fecha de recepción: 28 de abril de 2018. Fecha de aceptación: 5 de febrero de 2019.

${ }^{1}$ Universidad de Cantabria, Facultad de Ciencias, diegojm@unican.es orcid.org/0000-0002-4427-2724

2 Instituto Tecnológico Metropolitano, Colombia (Campus Robledo, Facultad de Ciencias Exactas y Aplicadas) franciscocordoba@itm.edu.co orcid.org/0000-0002-3371-3643 
en este contexto que permita identificar las creencias, y como consecuencia estudiar su influencia en el aprendizaje de las matemáticas.

Palabras clave: Creencias sobre las matemáticas, Educación secundaria, Cuestionario, Análisis factorial

\begin{abstract}
This article presents the adaptation and validation of the MRBQ (Mathematics-Related Beliefs Questionnaire) to the Colombian context. The MRBQ is an instrument for assessing student mathematics-related beliefs, originally developed at the University of Leuven (Belgium) and validated in several countries. The instrument was administered to 670 students, from public high schools in Medellín (Colombia). The analyses revealed that these student mathematics-related beliefs are better represented with a ten-factor model, rather than with a four-factor model as suggested in previous studies of the MRBQ. The 10 factors explain more faithfully the original theoretical model and account for a variance of $56.34 \%$, higher than that obtained in previous research. These results not only suggest significant improvements of the MRBQ, but also provide the basis for developing a reliable tool to assess beliefs in the Colombian context. To what these authors know, a consistent instrument has not been designed yet in this context for identifying beliefs, and consequently studying their influence on the learning of mathematics.
\end{abstract}

Keywords: Mathematics-related beliefs, Secondary education, Questionnaire, Factor analysis

\title{
INTRODUCCIÓN
}

Este artículo se centra en investigar las creencias sobre las matemáticas que tienen los estudiantes de educación secundaria en centros públicos de la ciudad de Medellín, Colombia. En Colombia el estudio sobre el aprendizaje de las matemáticas se ha tratado generalmente desde aspectos cognitivos (López, Hederich-Martínez y Camargo, 2012; Toro-Carvajal, Ortíz-Álvarez, Jiménez-García y Agudelo-Calle, 2012; Arévalo y González, 2013), meta-cognitivos (Zambrano, 2008; Iriarte, 2011; Santos y Lozada, 2013) y curriculares (Gómez, 2002; Murcia y Henao, 2015; Ángulo, Reyes, Triana y Aristizábal, 2016), sin considerar las 
dimensiones afectivas, ni el papel que juegan las creencias en el aprendizaje de esta materia. El objetivo del presente estudio es adaptar y validar un cuestionario como primer paso en el análisis sistemático y riguroso de las creencias sobre las matemáticas de los estudiantes colombianos. En particular se pretende adaptar y validar la versión del MRBQ (Mathematics-Related Beliefs Questionnaire) refinada por Diego-Mantecón (2013), que ha sido evaluada en diferentes contextos socio-culturales.

El estudio de las creencias se hace necesario cuando se busca comprender las causas de ciertas acciones que pueden conducir al fracaso o al éxito escolar en una materia tan significativa, pero a la vez tan estigmatizada como las matemáticas. En los últimos años se han atribuido distintos significados al término creencias; en este estudio se definen como un sistema de conocimiento subjetivo, personal y no siempre compartido (Pajares, 1992; Pehkonen, 1999; Parra, 2005; Diego-Mantecón, 2012, 2013). Se construyen y desarrollan durante la vida como fruto de las experiencias personales y de las interacciones con el entorno (Pehkonen y Pietilä, 2003), sirviendo a su vez como filtros a través de los que se procesan nuevas experiencias e informaciones. Estas nuevas experiencias se incorporan a un sistema de creencias ya existente, ayudando a consolidar o modificar creencias previas (Beck, 1976; Knapp y Beck, 2008). Las creencias pueden además mantenerse con diferentes grados de convicción, no siendo necesariamente compartidas y por lo tanto disputables (Hidalgo, Maroto y Palacios, 2015). De acuerdo con Diego-Mantecón (2012) las creencias anteceden a las actitudes, determinando y condicionando nuestras acciones en respuesta a determinadas situaciones y contextos.

\section{CREENCIAS SOBRE LAS MATEMÁTICAS, CONCEPTUALIZACIÓN Y EVALUACIÓN}

A continuación, se detalla la influencia de las creencias en el aprendizaje de las matemáticas, así como su conceptualización a la hora de estudiarlas. Se presentan también los instrumentos existentes para su evaluación, destacando como herramienta principal el MRBQ. 


\section{LA INFLUENCIA DE LAS CREENCIAS EN EL APRENDIZAJE DE LAS MATEMÁTICAS}

En la actualidad existe un consenso sobre la importancia de las creencias en el aprendizaje de las matemáticas (Bofah y Hannula, 2016; De Corte, 2015). Kilpatrick, Swafford y Findell (2001) afirman que en el 'desarrollo de la competencia matemática' intervienen cinco componentes principales; una de ellas es la 'disposición productiva' que comprende las creencias sobre las matemáticas, la motivación por su aprendizaje y la autoeficacia. Las creencias sobre las matemáticas funcionan como principios rectores que influyen en la toma de decisiones, pudiendo utilizarse para predecir acciones o conductas concretas (Schoenfeld, 2012; Martínez Padrón, 2013; Skott, 2015). Éstas determinan por lo tanto la forma en la que los estudiantes participan en el aprendizaje (Schoenfeld 1988; Op't Eynde, De Corte y Verschaffel, 2002), influyendo así en su rendimiento académico (House, 2006; De Corte, 2015; Diego-Mantecón y Córdoba-Gómez, 2018). En concreto, Pehkonen y Törner (1999) señalan que las creencias son necesarias para entender cómo los estudiantes plantean un problema, ya que éstas pueden influir en las técnicas de resolución empleadas, así como en el tiempo y esfuerzo invertido. Aunque la enseñanza de técnicas de resolución y métodos heurísticos son necesarios, podrían no ser suficientes para lograr un aprendizaje efectivo (Diego-Mantecón, 2012) ya que las creencias son "elementos constitutivos importantes del aprendizaje" (Op't Eynde et al., 2002, p.14).

McLeod (1994) sugiere que las experiencias en clases tradicionales conducen a desarrollar creencias negativas sobre las matemáticas y en concreto sobre la resolución de problemas. Por ejemplo, la creencia 'las matemáticas son algo procedimental que consiste en aplicar reglas' dificulta la toma de decisiones y el desarrollo de estrategias en la resolución de problemas no rutinarios (Schoenfeld, 1982; Kloosterman y Stage, 1992). El estudiante que considera las matemáticas como una asignatura puramente procedimental tiende, además, a convertirse en un aprendiz pasivo, poniendo más énfasis en la memorización que en la comprensión (Pehkonen y Törner, 1999). De manera similar, aquel que considera las matemáticas aburridas o poco atractivas difícilmente se involucra de forma activa en esta materia. Por ejemplo, la creencia 'las matemáticas son una asignatura aburrida' está inversamente relacionada con el tiempo que el estudiante dedica a esta materia (Hannula, 2006; Kislenko, Grevholm y Lepik, 2007). Por el contrario, el individuo que encuentra las matemáticas interesantes y aplicables en diferentes contextos es proclive a 
esforzarse más y a trabajar de forma constante (Pehkonen y Törner, 1999; Prendergast et al., 2018). Así mismo, los estudiantes que creen en su capacidad para resolver una situación matemática concreta tienden a perseverar hasta encontrar la solución (Bandura, 1999; Schunk y Pajares, 2002).

\section{CONCEPTUALIZACIÓN DE LAS CREENCIAS}

A pesar de los numerosos trabajos que existen en educación matemática sobre el estudio de las creencias (v.g. Kloosterman y Stage, 1992; Furinghetti y Pehkonen, 2002; Op't Eynde y De Corte, 2003; Lomas, Grootenboer y Attard, 2012; Diego-Mantecón, 2013; Andrews y Diego-Mantecón, 2015) no se ha establecido un consenso a la hora de definirlas. Furinghetti y Pehkonen (2002), después de analizar las definiciones utilizadas por diferentes expertos a nivel mundial, concluyen que no hay unanimidad y que una caracterización universal podría no ser posible. Diego-Mantecón (2012), tras una extensa revisión de la literatura, confirma que no existe tal consenso y señala, además, que a menudo no se hace un intento por definirlas o ponerlas en contexto con otras variables relacionadas, como las actitudes o las concepciones. Hannula (2004) señala que en la mayoría de los casos no se definen directamente, sino que se describen algunas de sus características para delimitarlas.

Las creencias se caracterizan, por ejemplo, por ser una forma de conocimiento subjetivo, que se mantiene con diferentes grados de convicción y consciencia, y por estar compuestas por elementos afectivos, evaluativos y sociales (Abelson, 1979; Pajares, 1992; Vila y Callejo, 2005; Gómez-Chacón, Op’t Eynde y De Corte, 2006). Son afectivas en el sentido de que están influenciadas por nuestras emociones (Pajares, 1992); evaluativas al ser aceptadas como verdaderas o falsas y servir como guía para el pensamiento (Villoro, 2006), y sociales al estar determinadas por el contexto en el que vivimos (Gómez-Chacón et al., 2006). Como resultado de lo anterior, las creencias se definen en este artículo como un sistema de conocimiento subjetivo y no siempre compartido (Pajares, 1992; Parra, 2005; Diego-Mantecón, 2012, 2013), que se modifica como consecuencia de las interacciones con el entorno (Pehkonen y Pietilä, 2003). 


\section{Modelos propuestos para el estudio de las creencias}

Con anterioridad a los 80 , las creencias sobre las matemáticas se estudiaban por lo general de forma separada (Op’t Eynde et al., 2002; Diego-Mantecón, 2012, 2013). Algunos autores estudiaron por ejemplo las 'creencias epistemológicas' (Schommer, Crouse y Rhodes, 1992; Schommer-Aikins, 2002); otros investigaron las 'creencias sobre las matemáticas como disciplina' (Schoenfeld, 1989); y otros analizaron las 'creencias del individuo en relación con las matemáticas' (Bandura, 1993; Pajares y Miller, 1994). A partir de la segunda mitad de los 80 se propusieron modelos que integran diferentes dimensiones. Frank (1985) planteó un modelo de cinco dimensiones, creencias acerca de: (1) la habilidad para estudiar matemáticas, (2) las matemáticas como disciplina, (3) el origen del conocimiento matemático, (4) la resolución de problemas, y (5) la enseñanza y aprendizaje de las matemáticas. A diferencia de Frank (1985), Underhill (1988) no consideró las creencias acerca de 'la resolución de problemas', y separó la dimensión 'enseñanza y aprendizaje de las matemáticas' en dos, estableciendo cuatro dimensiones: (1) las matemáticas como disciplina, (2) el aprendizaje, (3) la enseñanza, y (4) el contexto social.

McLeod (1992) propuso otro modelo de cuatro dimensiones, creencias acerca de: (1) la naturaleza de las matemáticas (incluyendo las matemáticas como disciplina y su aprendizaje), (2) uno mismo en relación con las matemáticas, (3) su enseñanza, y (4) el contexto social. Kloosterman (1996), en un modelo más reducido, sugirió solo dos dimensiones: creencias acerca de (1) las matemáticas y (2) de cómo aprenderlas. Esta segunda dimensión incluye varias sub-dimensiones; una de ellas, creencias acerca del aprendizaje, es considerada una dimensión principal en el modelo de Pehkonen (1995) que incluye cuatro dimensiones: (1) las matemáticas como disciplina, (2) uno mismo en relación con las matemáticas, (3) su enseñanza y (4) aprendizaje. Op’t Eynde et al. (2002) trataron de integrar los modelos anteriores en tres dimensiones interrelacionadas, que a su vez se dividen en 13 sub-dimensiones. Diego-Mantecón (2006) ilustró este modelo en una tabla proporcionando ejemplos concretos para cada sub-dimensión (ver tabla 1). 
Tabla 1. Modelo teórico de Op’t Eynde ejemplificado por Diego-Mantecón (2006, p.13)

\section{Creencias acerca de la educación matemática}

1.1 Creencias sobre las matemáticas como asignatura (v.g. Las matemáticas son básicamente cálculos, fórmulas y procedimientos)

1.2 Creencias sobre el aprendizaje matemático y la resolución de problemas (v.g. El aprendizaje de las matemáticas consiste principalmente en memorizar)

1.3 Creencias sobre la enseñanza de las matemáticas (v.g. El trabajo en grupo facilita el aprendizaje de las matemáticas)

\section{Creencias sobre uno mismo en relación con las matemáticas}

2.1 Creencias sobre la orientación intrínseca a la meta (v.g. Prefiero tareas matemáticas en las que tengo que esforzarme)

2.2 Creencias sobre la orientación extrínseca a la meta (v.g. Solo estoy satisfecho cuando obtengo una buena calificación en matemáticas)

2.3 Creencias sobre el valor de la tarea (v.g. Creo que las matemáticas que realizo en clase me serán útiles en el futuro)

2.4 Creencias sobre autocontrol (v.g. Si estudio de manera apropiada, puedo aprender los contenidos que me enseñan en matemáticas)

2.5 Creencias sobre autoeficacia

(v.g. Puedo entender incluso los conceptos más difíciles en matemáticas)

\section{Creencias acerca del contexto de la clase}

3.1 Creencias acerca del rol y la actuación del profesor 3.1.1 Creencias sobre la dimensión cognitiva del profesor (v.g. Nuestro profesor muestra paso a paso cómo debemos resolver un problema matemático)

3.1.2 Creencias sobre la dimensión motivacional del profesor (v.g. Nuestro profesor realmente quiere que disfrutemos aprendiendo cosas nuevas)

3.1.3 Creencias sobre la dimensión afectiva del profesor (v.g. A nuestro profesor le importa cómo nos sentimos en las clases de matemáticas)

3.2 Creencias acerca del rol y actuación del estudiante (v.g. Pregunto a mi profesor de matemáticas cuando no entiendo algo)

3.3 Creencias acerca de las normas y prácticas socio-matemáticas en la clase (v.g. Estoy siempre atento cuando mi profesor de matemáticas explica) 
En una revisión más actual Jankvist $(2009,2015)$ amplía el modelo de Op’t Eynde et al. (2002) proponiendo una cuarta dimensión 'creencias sobre las matemáticas como disciplina' en la que considera cuestiones sobre su origen y evolución; por ejemplo, si las matemáticas fueron descubiertas o inventadas.

\section{Instrumentos de evaluación de las creencias: el MRBQ}

Varios de los modelos anteriores se han evaluado mediante estudios cuantitativos y en concreto con cuestionarios o escalas Likert. El modelo de cuatro dimensiones propuesto por McLeod (1992) fue por ejemplo evaluado mediante el instrumento de Lazim, Abu y Wan (2004). De forma similar, la consistencia del modelo de Op't Eynde et al. (2002) fue verificada mediante el instrumento MRBQ (Mathematics-Related Beliefs Questionnaire) diseñado por Op't Eynde y De Corte (2003), y que evalúa 11 de sus 13 sub-dimensiones. ${ }^{3}$ El MRBQ es el instrumento de evaluación de las creencias que mayor fiabilidad ha mostrado en la última década. Se ha refinado en varios estudios, mostrando consistencia con estudiantes de diferentes contextos socioculturales incluidos España (Gómez-Chacón et al., 2006); España e Inglaterra (Diego-Mantecón, Andrews y Op’t Eynde, 2007; Diego-Mantecón, 2013); Inglaterra, Eslovaquia y España (Andrews, Diego-Mantecón, Vankúš y Op’t Eynde, 2011); España, Inglaterra, Eslovaquia e Irlanda (Diego-Mantecón y Andrews, 2008), y más recientemente Ecuador (De Corte, 2015). La continua evaluación del cuestionario en diferentes contextos le confiere más validez que otros instrumentos que han sido utilizados sólo en estudios puntuales.

El cuestionario original de 58 ítems fue testado por primera vez con 365 estudiantes flamencos (Op't Eynde y De Corte, 2003). Tras un análisis factorial con rotación ortogonal se forzó la extracción de cuatro factores, porque según sus autores este era el modelo que posibilitaba una mejor interpretación de los datos. Los cuatro factores se etiquetaron como: (1) Rol y actuación del profesor, (2) Importancia y competencia en matemáticas, (3) Las matemáticas como una actividad social, y (4) Las matemáticas como un dominio de excelencia. El

\footnotetext{
3 Las sub-dimensiones 3.2 'Creencias acerca del rol y actuación del estudiante en la clase' y 3.3 'Creencias acerca de las normas y prácticas socio-matemáticas en la clase' presentadas en la Tabla 1 no fueron consideradas en el MRBQ original, al no ser exploradas en profundidad en el modelo teórico (Op't Eynde et al., 2002).
} 
análisis explicó una varianza de 38.3\% y generó unas alfas de Cronbach de 0.92 , $0.89,0.65$ y 0.69 , para cada uno de los cuatro factores. Posteriormente, el MRBQ se validó en otros contextos internacionales, con las correspondientes adaptaciones. En estos nuevos análisis también se forzó la extracción de cuatro factores para facilitar la comparación de resultados con el estudio original. Se destacan en particular los estudios de Diego-Mantecón (2013) y De Corte (2015) realizados en España y Ecuador respectivamente. Como muestra la tabla 2, los análisis de Diego-Mantecón y De Corte explicaron una varianza de 39.4\% y de $46.8 \%$ respectivamente, superando en ambos casos la varianza reportada en el estudio original. La tabla 2 muestra también las variaciones en los valores de fiabilidad en cada factor, así como el refinamiento en el etiquetado para los tres estudios.

Tabla 2. Estudios anteriores del MRBQ. Factores, fiabilidad y varianza

\begin{tabular}{|c|c|c|c|c|c|}
\hline \multicolumn{2}{|l|}{$\begin{array}{c}\text { Op't Eynde y De Corte } \\
(2003)\end{array}$} & \multicolumn{2}{|l|}{$\begin{array}{c}\text { Diego-Mantecón } \\
\text { (2013) }\end{array}$} & \multicolumn{2}{|l|}{$\begin{array}{l}\text { De Corte } \\
(2015)\end{array}$} \\
\hline Factor & $\alpha$ & Factor & $\alpha$ & & $\alpha$ \\
\hline Rol y actuación del profesor & 0.92 & $\begin{array}{l}\text { Profesor como facilita- } \\
\text { dor del aprendizaje }\end{array}$ & 0.93 & $\begin{array}{l}\text { Rol y actuación del } \\
\text { profesor }\end{array}$ & 0.85 \\
\hline $\begin{array}{l}\text { Importancia y competencia } \\
\text { en matemáticas }\end{array}$ & 0.89 & Autoeficacia & 0.89 & $\begin{array}{l}\text { Significado y com- } \\
\text { petencia en mate- } \\
\text { máticas }\end{array}$ & 0.89 \\
\hline $\begin{array}{l}\text { Las matemáticas como una } \\
\text { actividad social }\end{array}$ & 0.65 & $\begin{array}{l}\text { Relevancia de las } \\
\text { matemáticas en la vida } \\
\text { real }\end{array}$ & 0.82 & $\begin{array}{l}\text { Las matemáticas } \\
\text { como una materia } \\
\text { que se puede } \\
\text { aprender }\end{array}$ & 0.79 \\
\hline $\begin{array}{l}\text { Las matemáticas como un } \\
\text { dominio de excelencia }\end{array}$ & 0.69 & $\begin{array}{l}\text { Las matemáticas como } \\
\text { una necesidad funcio- } \\
\text { nal de la vida escolar }\end{array}$ & 0.78 & $\begin{array}{l}\text { Las matemáticas } \\
\text { como una necesi- } \\
\text { dad funcional en la } \\
\text { vida escolar }\end{array}$ & 0.79 \\
\hline Varianza Explicada & $38.3 \%$ & & $39.4 \%$ & & $46.8 \%$ \\
\hline
\end{tabular}


Conviene resaltar que el estudio de Diego-Mantecón (2013), a diferencia de los otros estudios, identificó sub-factores en dos de los cuatro factores. Mediante un segundo análisis factorial Diego-Mantecón encontró que el factor 3 identificado como 'Relevancia de las matemáticas en la vida real' se subdivide en dos sub-factores: 'Aplicación de las matemáticas en la vida real' e 'Importancia de una comprensión flexible y profunda de las matemáticas'; y el factor 4 'Las matemáticas como una necesidad funcional de la vida escolar' está formado por los sub-factores: 'Las matemáticas como asignatura inaccesible' y las 'Matemáticas como asignatura de destrezas mecánicas'. De acuerdo con lo anterior, Diego-Mantecón identificó seis dimensiones de creencias, aportando de esta forma información más precisa a la estructura original de cuatro factores.

Lo descrito anteriormente muestra al MRBQ como uno de los instrumentos más utilizados y más fiables para evaluar las creencias de los estudiantes en diferentes contextos socio-culturales.

\section{METODOLOGÍA}

Como se ha indicado con anterioridad las creencias son una variable fundamental en el aprendizaje de las matemáticas, permitiendo identificar factores que influyen en este proceso. Disponer de un cuestionario como el MRBQ que evalúe de forma fiable las creencias acerca de las matemáticas se hace por lo tanto necesario. En concreto, este estudio tiene como objetivo proporcionar a la comunidad educativa una adaptación y validación del MRBQ al contexto colombiano, ya que en la actualidad no se conocen instrumentos que identifiquen con fiabilidad las creencias en este contexto.

Para evitar problemas de equivalencia conceptual y lingüística (Helms, 1992; Osborn, 2004) se tomó como base la versión de Diego-Mantecón (2013) que había sido traducida al español y validada en España. Además de adaptar los ítems para establecer equivalencia transcultural, éstos fueron también revisados para incrementar su validez de contenido (De Vellis, 2003; McQueen y Knussen, 2002,) y establecer su validez interna (Sarantakos, 2013; Cohen, Manion y Morrison, 2003), aspectos no considerados en estudios anteriores de este instrumento. Para incrementar la validez de los ítems en términos de contenido, y asegurar que evaluaban los aspectos pretendidos por los investigadores (McQueen y Knussen, 2002), éstos fueron revisados por un comité de expertos. En este sentido varios de los ítems, en particular aquellos que incluían más de una idea, se 
eliminaron o reformularon al conducir a respuestas sesgadas. Por ejemplo, el ítem 'Las matemáticas son una asignatura necesaria y que vale la pena' se reformuló de la siguiente manera 'Las matemáticas son una asignatura necesaria'.

La validez interna se refiere al grado en el que el diseño de los instrumentos puede afectar a los resultados del estudio (Sarantakos, 2013). En este trabajo se incrementó la validez interna de los ítems verificando que estos eran comprensibles por los sujetos a investigar, tal y como sugieren Cohen et al. (2003). Para ello se administró inicialmente el cuestionario a un grupo de 30 estudiantes de secundaria, con características similares a las de la muestra principal. Varios ítems que resultaron confusos para los alumnos fueron refinados, mejorando la redacción y sintetizando la información. Durante este proceso también se reformularon aquellos ítems que en el instrumento original estaban orientados negativamente, por ser este un factor que reduce la calidad de las respuestas y afecta la consistencia interna de las escalas (Suárez-Álvarez et al., 2018). Por ejemplo, el ítem 'A mi profesor no le preocupa cómo nos sentimos en clase' se reformuló 'A mi profesor le preocupa cómo nos sentimos en clase'.

Una vez realizados los ajustes anteriores, el cuestionario fue aplicado a una muestra no aleatoria de 670 estudiantes (357 hombres y 313 mujeres) a la que los investigadores tenían acceso. Los participantes eran de edades comprendidas entre los 14 y 15 años, siguiendo la línea de los estudios originales. Esta edad se corresponde en Colombia con el noveno curso de educación secundaria. Los estudiantes pertenecían a seis centros públicos situados en la periferia de la ciudad de Medellín y representativos de un nivel socioeconómico bajo, correspondiéndose con los niveles 1 y 2 de estratificación en una escala de 6 niveles, donde 1 es el nivel más bajo. El cuestionario fue administrado directamente por uno de los investigadores para evitar sesgos en la recogida de datos.

\section{ANÁLISIS DE DATOS Y RESULTADOS}

Para evaluar la validez del instrumento en el contexto colombiano se aplicó un análisis factorial con rotación ortogonal, como en los estudios originales. Antes de computar el análisis factorial se realizó un escrutinio de los datos para evaluar el grado de adecuación de este análisis a la muestra. Se computó una matriz de correlaciones para eliminar aquellos ítems que correlacionaban muy bajo (con valores inferiores a 0.20 ) o muy alto (con valores superiores a 0.90 ), y que 
por lo tanto no son recomendables a la hora de aplicar análisis factorial (Field, 2009). También se computó el test de adecuación muestral de Kaiser Meyer-Olkin (KMO) y la prueba de esfericidad de Bartlett que arrojó un valor de 0.921 con una $p<0.00005$, lo que sugirió que los datos eran adecuados para aplicar análisis factorial (Field, 2009).

A diferencia de los estudios anteriores realizados con el MRBQ no se forzó la extracción de cuatro factores, sino que se consideró el criterio de Kaiser (1960) y el test de Cattell (1978) para determinar los factores a extraer. El criterio de Kaiser recomienda extraer el número de factores correspondientes a los valores propios mayores que uno, y el test de Cattell aquellos que indica el punto de inflexión del gráfico de sedimentación. En este caso, se optó por la extracción de 10 factores recomendados por el criterio de Kaiser ya que permitían una interpretación más completa de los datos. Los 10 factores extraídos explican una varianza de 56.34\%. En la tabla 3 se muestran los factores extraídos con sus respectivos ítems y cargas factoriales. 


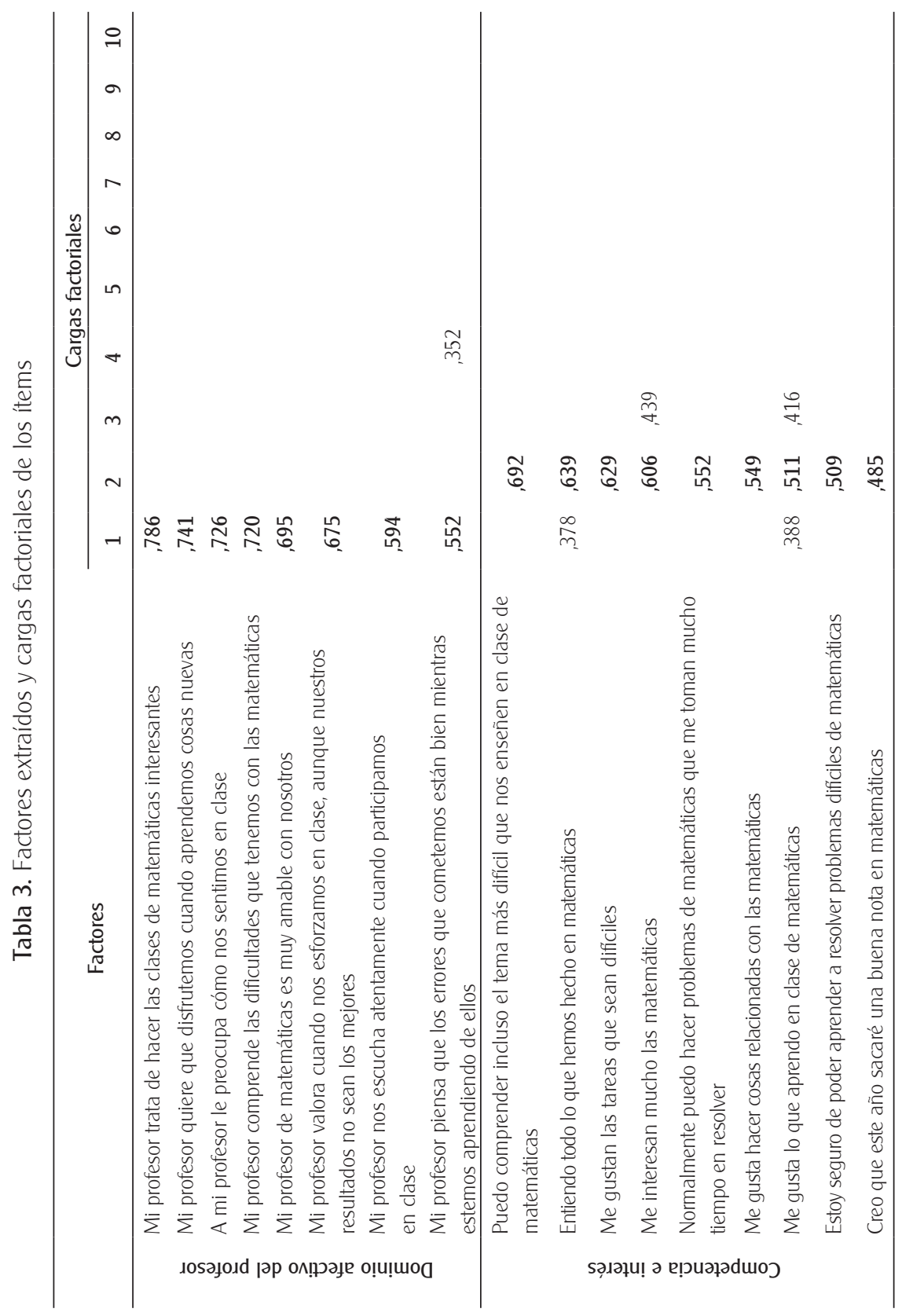




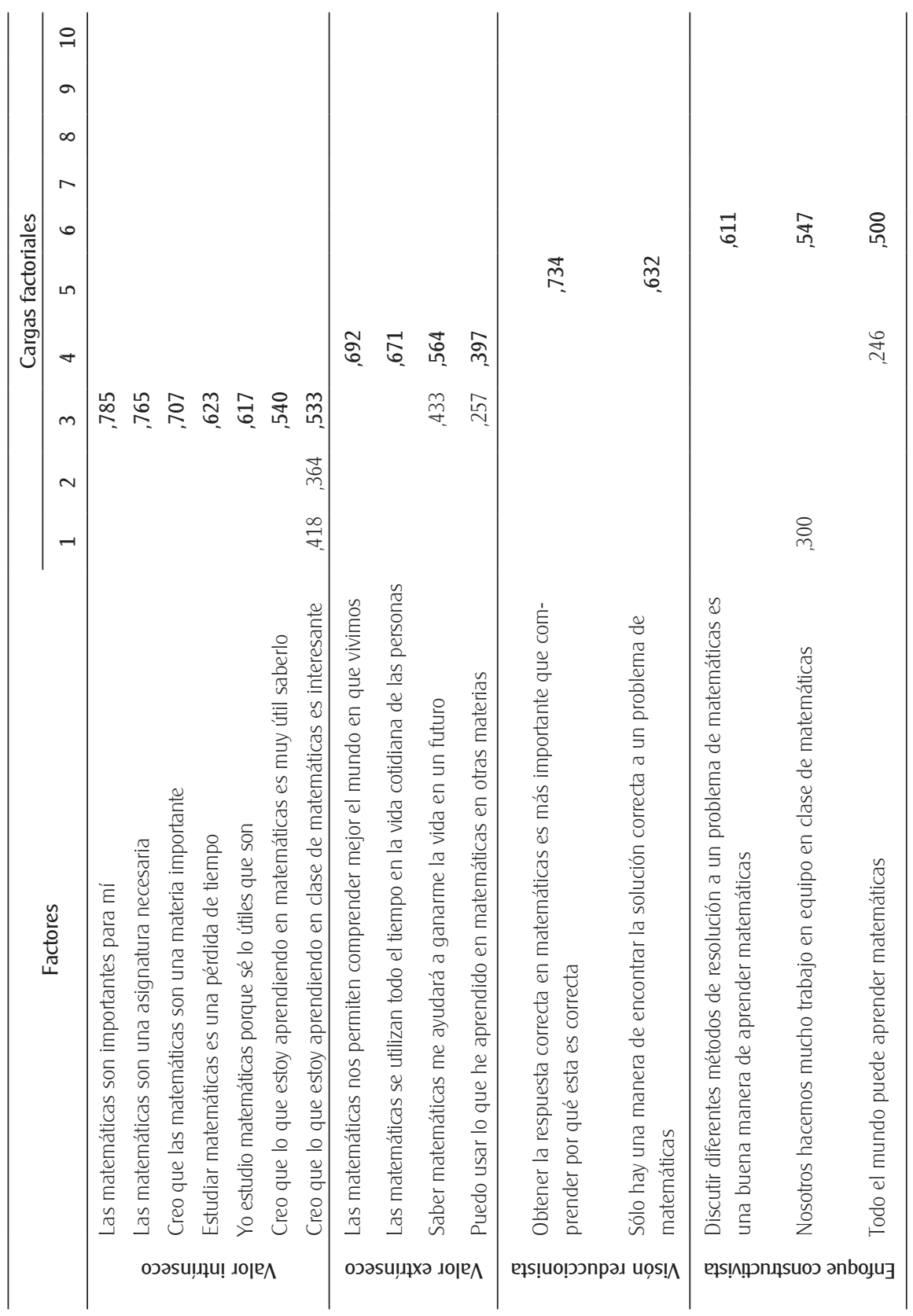




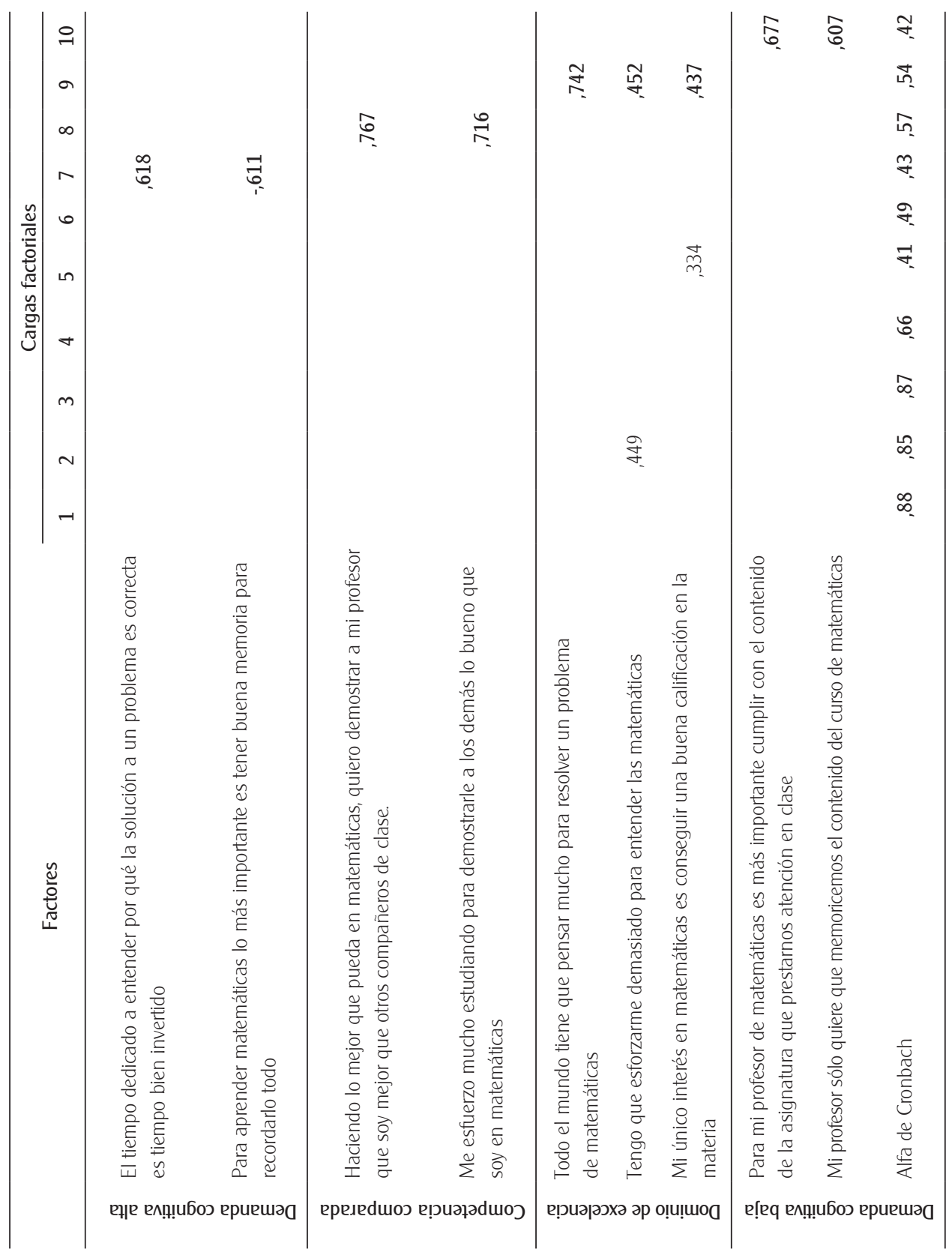


Para etiquetar los factores extraídos se consideraron los ítems con mayor carga factorial en cada columna; de acuerdo con Field (2009) y De Vellis (2003) estos son los que mejor representan el significado de los factores. El factor 1 se etiquetó como Dominio afectivo del profesor al incluir ítems con expresiones relacionadas con las emociones del profesor, durante la interacción con los alumnos y su aprendizaje, tales como 'trata de hacer las clases interesantes' o 'quiere que disfrutemos'. El factor 2 hace alusión a la Competencia e interés por las matemáticas ya que incluye ítems relacionados con la percepción de uno mismo sobre su capacidad a la hora de trabajar las matemáticas (v.g. 'Puedo comprender incluso el tema más difícil') e ítems relacionados con el interés o gusto por esta materia (v.g. 'Me interesan las matemáticas'). El factor 3 se etiquetó Valor intrínseco de las matemáticas al aparecer en éste expresiones relacionadas con el valor de las matemáticas en sí mismas (v.g. 'Las matemáticas son importantes para mí) y no por un valor externo que se pueda obtener de su estudio o aprendizaje. El factor 4 se identificó Valor extrínseco de las matemáticas al contener ítems tales como 'se utilizan en la vida cotidiana' o 'nos permiten comprender mejor el mundo en el que vivimos' relacionados con la utilidad de las matemáticas como herramienta para resolver situaciones reales y comprender nuestro entorno. El factor 5 se relacionó con una Visión reduccionista de las matemáticas; expresiones como 'obtener la respuesta correcta es lo más importante' o 'sólo hay una manera de encontrar la solución correcta' evidencian un enfoque práctico y limitado de las matemáticas, percibiéndose éstas en términos de resultados a los que se llega por una sola vía.

El factor 6 se relaciona con un Enfoque constructivista de las matemáticas. Ítems como 'Discutir diferentes métodos de resolución es una buena manera de aprender' o 'Hacemos mucho trabajo en equipo' entroncan con una enseñanza constructivista en la que se enfatiza el proceso de resolución sobre el propio resultado, y se valora el aprendizaje colaborativo. El factor 7 hace referencia a una Demanda cognitiva alta al aparecer en éste ítems que resaltan la importancia de los procesos de comprensión (v.g. 'Entender por qué una solución es correcta es tiempo bien invertido') frente a los de memorización (v.g. Tener buena memoria no es lo más importante para aprender matemáticas'). El factor 8, Competencia comparada, incluyó dos ítems que reflejan el deseo por mostrar una alta capacidad y reconocimiento frente a los demás: 'Quiero demostrar a mi profesor que soy mejor que otros' y 'Me esfuerzo estudiando para demostrar lo bueno que soy'. El factor 9 se designó Dominio de excelencia al representar las matemáticas como accesibles sólo para aquellos capaces de pensar y esforzarse mucho (v.g. Todo el mundo 
tiene que pensar mucho para resolver un problema de matemáticas'). Finalmente, el factor 10 se etiquetó Enfoque tradicionalista de las matemáticas ya que hace alusión a una enseñanza centrada en los contenidos y su memorización. Así lo reflejan ítems tales como: 'Mi profesor sólo quiere que memoricemos el contenido' o 'Para mi profesor es más importante el contenido de la asignatura que prestarnos atención'.

Una vez extraídos y etiquetados los factores se aplicó un segundo análisis factorial en cada uno de ellos para asegurar su unidimensionalidad; posteriormente se computó el alfa de Cronbach para conocer su fiabilidad (Wells y Wollack, 2003). Como muestra la tabla 3, tres de los factores presentaron una consistencia interna alta (Field, 2009) con alfas superiores a 0.80, tres una consistencia media con valores entre 0.54 y 0.66 , y el resto una consistencia baja con valores entre 0.40 y 0.50 .

\section{DISCUSIÓN DE RESULTADOS}

Los resultados descritos anteriormente muestran que, si no se fuerza la extracción de factores y se atiende a los criterios de extracción, el MRBQ genera un modelo de 10 factores que se corresponde, en gran medida, con el modelo teórico propuesto por Op't Eynde et al. (2002). Estos 10 factores se alinean en concreto con las 11 sub-dimensiones de creencias descritas inicialmente en su modelo y no con las cuatro dimensiones que se han venido identificando en los estudios empíricos hasta el momento (ver por ejemplo Op't Eynde y De Corte, 2003; Gómez-Chacón et al., 2006; Diego-Mantecón, 2013; y De Corte, 2015). Estos resultados son significativos, ya que la identificación de los 10 factores permite un estudio más detallado de las creencias del estudiante, posibilitando análisis más precisos sobre su influencia en el aprendizaje.

Los resultados del presente estudio muestran que varias de las dimensiones inicialmente propuestas por Op't Eynde et al. (2002), y que emergieron en estudios anteriores bajo un mismo factor, ahora surgen separadas en factores diferentes. Por ejemplo, las dimensiones sobre 'la importancia de las matemáticas', 'la utilidad de las matemáticas' y 'la orientación extrínseca a la meta', consideradas entidades independientes en el modelo teórico, aparecen representadas en este estudio por los factores Valor intrínseco de las matemáticas', Valor extrínseco de las matemáticas' y 'Competencia comparada'. En estudios anteriores estas dimensiones emergieron repetidamente bajo un mismo factor: en los 
trabajos de Gómez-Chacón et al. (2006) y De Corte (2015) aparecieron bajo el factor 'Creencias sobre el significado y la competencia en matemáticas', y en el de Diego-Mantecón (2013) bajo el factor denominado 'Relevancia de las matemáticas en la vida real'. Forzar la extracción de cuatro factores ha provocado que diferentes dimensiones de creencias se aglutinen bajo un mismo factor. Este hecho ha impedido análisis pormenorizados de cada dimensión lo que ha dificultado conocer con precisión los tipos de creencias que influyen más en el rendimiento académico del estudiante. Contar con factores unidimensionales, como los obtenidos en el presente estudio, permitirá establecer relaciones entre los diferentes tipos de creencias, proporcionando información más precisa sobre su influencia en el aprendizaje de las matemáticas.

De acuerdo con lo anterior, el modelo de 10 factores es más explicativo que el modelo de cuatro y da cuenta de varias de las dimensiones subyacentes que componen el modelo teórico, lo que posibilitará comprender más ampliamente el espectro de creencias de los estudiantes. Estos resultados no se hubieran obtenido forzando la extracción de cuatro factores. Es importante destacar que el estudio de Diego-Mantecón (2013), que revelaba sub-factores a través de un segundo análisis factorial, ya sugería trabajar con factores unidimensionales. En este sentido, el presente estudio respalda el análisis de Diego-Mantecón (2013), en cuanto a la búsqueda de factores unidimensionales, y lo mejora en cuanto al hecho de no forzar la extracción de un determinado número y guiarse por criterios de extracción contrastados, como el de Kaiser (1960) y el de Cattell (1978), que resultan útiles en estudios de carácter exploratorio (Yong y Pearce, 2013). Como se ha descrito anteriormente, el análisis de Diego-Mantecón (2013) identificó seis dimensiones de creencias, después de aplicar el segundo análisis factorial, frente a las 10 obtenidas en el presente estudio.

Desde el punto de vista analítico y de fiabilidad del instrumento, los 10 factores extraídos explican una varianza de $56.34 \%$ superior a la de los cuatro factores descritos en estudios anteriores. Como se ha indicado previamente, los factores extraídos por Op't Eynde y De Corte (2003), Diego-Mantecón (2013) y De Corte (2015) explicaron varianzas de 38.3\%, 39.4\% y 46.8\% respectivamente. Importante, los 10 factores obtenidos en el presente estudio son unidimensionales; es decir, cada uno representa una única variable de creencias. Tres de ellos mostraron una consistencia interna alta con alfas de Cronbach superiores a 0.80 , lo que les aporta fiabilidad para ser utilizados en el contexto colombiano. Otras tres mostraron una consistencia media con valores entre 0.54 y 0.66, y el resto una consistencia baja con valores entre 0.40 y 0.50 . Estos últimos son 
susceptibles de ser refinados en futuros estudios para mejorar su fiabilidad, pero son factores potencialmente consistentes ya que apenas incluyen dos ítems. Por ejemplo, el factor Visión reduccionista de las matemáticas' que generó una alfa de 0.49, está conformado únicamente por dos ítems 'Obtener la respuesta correcta en matemáticas es más importante que entender por qué ésta es correcta' y 'Sólo hay una manera de encontrar la solución correcta a un problema de matemáticas'. Estos ítems arrojaron unas cargas factoriales aceptables de 0.734 y 0.632 respectivamente, sugiriendo que la fiabilidad del factor se puede mejorar en investigaciones posteriores con la inclusión de nuevos ítems que ayuden a conformar la variable que representan.

Como muestra la tabla 3, la mayoría de los factores con una consistencia media o baja son aquellos que se han generado a partir de dos o tres ítems máximo. Este reducido número en cada factor se debe a que las escalas originales -diseñadas por Op't Eynde y De Corte (2003) para validar su modelo teórico- se han ido distorsionando tras las continuas adaptaciones del MRBQ. Inicialmente el MRBQ constaba de 58 ítems, este número se redujo a 40 en el primer estudio de Op't Eynde y De Corte (2003) con estudiantes flamencos, después de forzar la extracción de cuatro factores. Los estudios posteriores se centraron, por lo general, en adaptar o mejorar la fiabilidad de estos cuatros factores a un contexto particular, y no en perfeccionar las escalas originales encaminadas a evaluar las 11 variables del modelo teórico. Por ejemplo, Gómez-Chacón et al. (2006) añadieron cuatro ítems nuevos a los 40 resultantes del estudio de Op't Eynde y De Corte (2003) para evaluar las creencias de estudiantes españoles. De forma similar, Diego-Mantecón (2006) y Diego-Mantecón et al. (2007) incorporan 33 a los 40 existentes con el objetivo de mejorar la fiabilidad de los cuatro factores extraídos en investigaciones previas, y adaptar el instrumento al contexto eslovaco, inglés y español. Como en estudios previos, Diego-Mantecón $(2006,2013)$ forzó la extracción de cuatro factores para facilitar la comparación de datos.

En síntesis, los autores que han empleado el MRBQ se han centrado, por lo general, en mejorar la fiabilidad de los cuatro factores extraídos en el estudio de Op't Eynde y De Corte (2003), eliminando ítems originales y diseñando otros nuevos. Estos estudios han obviado las dimensiones de creencias inicialmente propuestas en el modelo teórico de Op't Eynde et al. (2002) y las escalas originales diseñadas para su evaluación (Op’t Eynde y De Corte, 2003). Esto explica que en el presente trabajo algunos de los 10 factores extraídos estén constituidos únicamente por dos ítems. La fiabilidad de estos factores es susceptible de aumentar al incluir nuevos ítems y mejorar las escalas originales que representan. 


\section{CONCLUSIONES}

Como se ha descrito inicialmente, el objetivo fundamental del presente estudio era adaptar y validar la versión española del MRBQ al contexto colombiano, para ofrecer a la comunidad educativa un instrumento fiable que posibilite identificar y analizar las creencias de los estudiantes. Los resultados mostraron cierto grado de consistencia del instrumento para su uso en el contexto colombiano, sirviendo este como base para el desarrollo de uno más refinado que permita evaluar las creencias con mayor fiabilidad.

Este trabajo reveló, a diferencia de estudios anteriores, que el MRBQ replica en cierto grado el modelo teórico inicialmente propuesto por Op't Eynde et al. (2002) y que había sido obviado, al menos parcialmente, en otras investigaciones. En particular, los resultados revelan que el MRBQ genera, en el contexto colombiano, un modelo de 10 factores -que permite una mayor comprensión e interpretación de las creencias de los estudiantes- y no uno de cuatro como el que se había obtenido reiteradamente en evaluaciones anteriores. Estos 10 factores representan más fielmente el modelo teórico original; cada uno de ellos está bien definido y ha mostrado ser unidimensional, lo que constituye un primer paso hacia la construcción de un instrumento válido para el estudio de las creencias acerca de las matemáticas en Colombia.

Es importante resaltar que algunos de los factores identificados se generaron a partir de sólo dos ítems y mostraron un valor de fiabilidad medio-bajo. Es, por lo tanto, necesario llevar a cabo nuevos estudios encaminados a refinar y completar las escalas que representan estos factores, mejorando la edición de algunos ítems e incorporando otros nuevos que ayuden a incrementar la consistencia de los factores. La realización de estos estudios ayudará a la construcción de un instrumento más sólido, que facilitará análisis más rigurosos y como consecuencia resultados más precisos sobre las creencias y su influencia en el aprendizaje de las matemáticas.

\section{AGRADECIMIENTOS}

Este trabajo se ha elaborado bajo el marco del proyecto de investigación EDU2017-84979-R del gobierno de España. También ha recibido apoyo del programa de Investigación e Innovación Horizon 2020 de la Unión Europea, bajo el acuerdo Horizon 2020- Seac-2015- 1-710577. Durante la realización de 
este trabajo, Francisco Javier Córdoba-Gómez era estudiante de doctorado en la Universidad de Cantabria (España).

\section{REFERENCIAS}

Abelson, P. (1979). Differences between belief and knowledge systems. Cognitive Science, 3, 355- 366.

Andrews, P. y Diego-Mantecón, J. M. (2015). Instrument adaptation in cross-cultural studies of students' mathematics-related beliefs: Learning from healthcare research. Compare: A Journal of Comparative and International Education, 45(4), 545-567.

Andrews, P., Diego-Mantecón, J. M., Vankúš, P. y Op’t Eynde, P. (2011). Construct consistency in the assessment of students' mathematics-related beliefs: a three-way cross-sectional pilot comparative study. Acta Didactica Universitatis Comenianae Mathematics, 11, 1-25.

Angulo, L., Reyes, A., Triana, K. y Aristizábal, A. (2016). Análisis y recomendaciones para el cambio del currículo en la escuela: El programa PISA, los estándares básicos de competencias y los planes de estudio de matemáticas. Revista Científica, Edición Especial, 34-35.

Arévalo, C. y González, O. (2013). La compresión y reflexión de los procesos cognitivos que se generan en las prácticas de estudiantes para profesor a la hora de demostrar en geometría. Revista Científica, Edición Especial, 27-31.

Bandura, A. (1993). Perceived self-efficacy in cognitive development and functioning. Educational Psychologist, 28, 117-148.

Bandura, A. (1999). Social cognitive theory: An aggentic perspective. Asian Journal of Social Psychology, 2, 21-41.

Beck, A.T. (1976). Cognitive therapy and the emotional disorders. Madison: International Universities Press.

Bofah, E. y Hannula, M. (2016). Students' views on mathematics in single-sex and coed classrooms in Ghana. European Journal of Science and Mathematics Education, 4(2), 229-250.

Cattell, R.B. (1978). The scientific use of factor analysis in behavioral and life sciences. New York, NY: Plenum Press.

Cohen, L., Manion, L., y Morrison, K. (2003). Research methods in education. London: Routledge Falmer 
De Corte, E. (2015). Mathematics-related beliefs of Ecuadorian students of grades 8-10. International Journal of Educational Research, 72, 1-13.

De Vellis, R. F. (2003). Scale Development: Theory and Applications (2nd ed.). Thousand Oaks, CA: Sage Publications.

Diego-Mantecón, J. M. (2006). A comparison of secondary English and Spanish Students' conceptions about Mathematics. University of Cambridge: Faculty of Education.

Diego-Mantecón, J. M. (2012). Clarifying the field of student mathematics-related beliefs: developing measurement scales for 14/15-year-old students across Bratislava, Cambridgeshire, Cantabria, and Cyprus. Doctoral thesis. Faculty of Education, University of Cambridge.

Diego-Mantecón, J. M. (2013). Evaluación de un modelo de creencias transcultural para el aprendizaje de las matemáticas. Gaceta de la Real Sociedad Matemática Española, 16(3), 561-574.

Diego-Mantecón, J. M. y Andrews, P. (2008). Construct consistency in the assessment of students' mathematics-related beliefs: a four-way cross-sectional pilot study. 11th International Congress on Mathematical Education ICME. Monterrey, México.

Diego-Mantecón, J. M., Andrews, P. y Op’t Eynde, P. (2007). Mejora y evaluación de un cuestionario de creencias de matemáticas en función de nacionalidad, edad y sexo. En M. Camacho, P. Flores y P. Bolea (Eds.), Investigación en educación matemática, XI, 325-333.

Diego-Mantecón, J. M. y Córdoba-Gómez, F. (2018). Creencias acerca de las matemáticas de estudiantes colombianos: adaptación y validación de un cuestionario. Acta Latinoamericana de Matemática Educativa, 31(1), 418-424.

Field, A. (2009). Discovering statistics using SPSS. London: Sage Publications.

Frank, M. L. (1985). Mathematical beliefs and problem solving. Unpublished doctoral dissertation. Purdue University. University Microfilms International.

Furinghetti, F. y Pehkonen, E. (2002). Rethinking characterizations of beliefs. In. G.C. Leder, E. Pehkonen y G. Törner (Eds.), Beliefs: A Hidden Variable in Mathematics Education (pp. 39-57). Dordrecht, The Netherlands: Kluwer.

Gómez, P. (2002). Análisis didáctico y diseño curricular en matemáticas. Revista EMA, 7(3), 251-292.

Gómez-Chacón, I., Op’t Eynde, P. y De Corte, E. (2006). Creencias de los estudiantes de matemáticas. La influencia del contexto de clase. Enseñanza de las Ciencias. 24(3), 309- 324.

Hannula, M. (2004). Affect in mathematical thinking and learning. Doctoral thesis. Faculty of Education, University of Turku, Finland. 
Hannula, M. (2006). Motivation in mathematics: goals reflected in emotions. Educational Studies in Mathematics, 63, 165-178.

Helms, J. E. (1992). Why is there no study of cultural equivalence in standardized cognitive ability testing? American Psychologist, 47, 1083-1101.

Hidalgo, S., Maroto, A. y Palacios, A. (2015). Una aproximación al sistema de creencias matemáticas en futuros maestros. Educación Matemática, 27(1), 65-90.

House, J. D. (2006). Mathematics beliefs and achievement of elementary school students in Japan and the United States: Results from the Third International Mathematics and Science Study. The Journal of genetic psychology, 167(1), 31-45.

Iriarte, A. (2011). Desarrollo de la competencia resolución de problemas desde una didáctica con enfoque metacognitivo. Zona Próxima: Revista del Instituto de Estudios Superiores en Educación, (15), 2-21.

Jankvist, U. (2009). Using history as a 'Goal' in mathematics education (Tesis Doctoral thesis). Roskilde: IMFUFA, Roskilde University. No. 464 in Tekster fra IMFUFA.

Jankvist, U. (2015). Changing students' images of "mathematics as a discipline. Journal of Mathematical Behavior, 38, 41-56.

Kaiser, H. F. (1960). The application of electronic computers to factor analysis. Educational and Psychological Measurement, 20, 141-151.

Kilpatrick, J., Swafford, J. y Findell, B. (2001). Adding it up: Helping children learn mathematics. Washington, DC: National Academy Press.

Kislenko, K., Grevholm, B. y Lepik, M. (2007). Mathematics is important but boring: Students' beliefs and attitudes towards mathematics. In C. Bergsten, B. Grevholm, H. S. Masoval y F. Ronning (Eds.). Relating practice and research in mathematics education. Proceedings of NORMA 05, (pp. 349-360). Trondheim, Norway: Tapir Academic Press.

Kloosterman, P. y Stage, F. (1992). Measuring beliefs about mathematical problem solving. School Science and Mathematics, 92 (3), 109-115.

Kloosterman, P. (1996). Students' beliefs about knowing and learning mathematics: Implications for motivation. In M. Carr (Ed.), Motivation in Mathematics (pp. 131-156). Cresskill, NJ: Hampton Press.

Knapp, P. y Beck, A. T. (2008). Cognitive therapy: foundations, conceptual models, applications and research. Revista Brasileira de Psiquiatria, 30, S54-S64.

Lazim, M. A., Abu Osman, M. T. y Wan Salihin, W. A. (2004). The statistical evidence in describing the students' beliefs about mathematics. International Journal for Mathematics Teaching and Learning, 77.

Lomas, G., Grootenboer, P. y Attard, C. (2012). The affective domain and mathematics education. In B. Perry et al., (Eds.), Research in Mathematics Education in Australasia 2008-2011, (pp. 23-37). Rotterdam: Sense Publischers. 
López, O., Hederich-Martínez, C. y Camargo, A. (2012). Logro en matemáticas, autorregulación del aprendizaje y estilo cognitive. Suma Psicológica, 19(2), 39-50.

Martínez Padrón, O. (2013). Las creencias en la educación matemática. Educere, 17(57), 231-239.

McLeod, D. (1992). Research on affect in mathematics education: A reconceptualization. In D.A. Grouws (Ed.), Handbook of Research on mathematics Teaching and Learning, (pp. 575-598). New York: Macmillan.

McLeod, D. (1994). Research on Affect and Mathematics Learning in the JR-ME: 1970 to the Present. Journal for Research in Mathematics Education, 25 (6), 637-647.

McQueen, R. y Knussen, C. (2002). Research methods for social science: An introduction. Harlow: Pearson Education Limited.

Murcia, M. y Henao, J. (2015). Educación matemática en Colombia, una perspectiva evolucionaria. Entre Ciencia e Ingeniería, 9(18), 23-30.

Op't Eynde, P. y De Corte, E. (2003). Students' mathematics- Related belief systems: Design and analysis of questionnaire. Paper presented at the 2003 Annual Meeting of the American Educational Research Association, April 21-25, Chicago.

Op't Eynde, P., De Corte, E. y Verschaffel, L. (2002). Framing students' mathematics - related beliefs: A quest for conceptual clarity and a comprehensive categorization. In G.C. Leder, E. Pehkonen, y G.Törner (Eds.), Beliefs: A Hidden Variable in Mathematics Education? (pp. 13-37). Dordrecht, The Netherlands: Kluwer Academic Publishers.

Osborn, M. (2004). New methodologies for comparative research; Establishing 'constants' and 'contexts' in educational experience. Oxford Review of Education, 30(2), 265-285.

Pajares, F. (1992). Teachers' Beliefs and Educational Research: Cleaning Up a Messy Construct. Review of Educational Research, 62 (3), 307-332.

Pajares, F. y Miller, D. (1994). Role of self-efficacy and self-concept beliefs in mathematical problem solving: A path analysis. Journal of Educational Psychology, 86(2), 193-203.

Parra, H. (2005). Creencias matemáticas y la relación entre actores del contexto. Revista Latinoamericana de Investigación en Matemáticas Educativa, 8(1), 69-90.

Pehkonen, E. (1995). Pupils' View of Mathematics: Initial report for an international comparison project (Research Report 152). University of Helsinki: Department of Teacher Education.

Pehkonen, E. (1999). Beliefs as obstacles for implementing an educational change in problem solving. In E. Pehkonen y G. Törner (Eds.), Mathematical beliefs and their impact on teaching and learning of mathematics: Proceedings of the workshop in Oberwolfach, November 1999. (pp. 106-114). Duisburg, Germany: Gerhard Mercator Universität Duisburg.

Pehkonen, E. y Törner, G. (1999). Introduction to the abstract book for the Oberwolfach meeting on belief research. In E. Pehkonen y G. Törner (Eds.), Mathematical beliefs 
and their impact on teaching and learning of mathematics: Proceedings of the workshop in Oberwolfach, November 1999. (pp. 50-56). Duisburg, Germany: Gerhard Mercator Universität Duisburg.

Pehkonen, E. y Pietilä, A. (2003). On relationships between beliefs and knowledge in mathematics education. In Proceedings of the Congress of European Research in Mathematics Education CERME 3, Bellaria, Italy.

Prendergast, M., Breen, C., Bray, A., Faulkner, F., Carroll, B., Quinn, D. y Carr, M. (2018). Investigating secondary students' beliefs about mathematical problem-solving. International Journal of Mathematical Education in Science and Technology, 49(8), 1-16.

Santos, D. y Lozada, G. (2013). ¿Es posible hacer evidentes los procesos de meta-cognición en la resolución de problemas? Revista Científica, Edición Especial, 47-50.

Sarantakos, S. (2013). Social research. Hong Kong: Macmillan International Higher Education.

Schoenfeld, A. (1982). Expert and Novice Mathematical Problem Solving. Final Project Report and Appendices B-H. Washington, D.C.: National Science Foundation.

Schoenfeld A. (1988). When good teaching leads to bad results: the disasters of 'welltaught' mathematics: the disasters of 'well-taught' mathematics courses. Educational Psychologist, 23(2), 145-166.

Schoenfeld, A. (1989). Explorations of student's mathematical beliefs and behavior. Journal for Research in Mathematics Education, 20(4), 338- 355.

Schoenfeld, A. H. (2012). How we think: A theory of human decision-making, with a focus on teaching. In The Proceedings of the 12th International Congress on Mathematical Education (pp. 229-243). Springer International Publishing.

Schommer, M., Crouse, A. y Rhodes, N. (1992). Epistemological beliefs and mathematical text comprehension: Believing it is simple does not make I so. Journal of Educational Psychology, 84, 435-443.

Schommer-Aikins, M. (2002). An evolving theoretical framework for an epistemological belief system. In. B. K. Hofer y P.R. Pintrich (Eds.), Personal epistemology: The psychology of beliefs about knowledge and knowing (pp. 103-118). Mahwah, NJ: Erlbaum.

Schunk, D. H. y Pajares, F. (2002). The development of academic self-efficacy. In A. Wigfield y J. S. Eccles (Eds.), Development of achievement motivation (pp. 16-32). San Diego, CA: Academic Press.

Skott, J. (2015). Towards a participatory approach to 'beliefs' in mathematics education. In B. Pepin y B. Roesken-Winter (Eds.), From Beliefs to Dynamic Affect Systems in Mathematics Education. Exploring a Mosaic of Relationships and Interactions (pp. 3-23). Switzerland: Springer. 
Suárez-Álvarez, J., Pedrosa, I., Lozano, L. M., García-Cueto, E., Cuesta, M. y Muñiz, J. (2018). Using reversed items in likert scales: A questionable practice. Psicothema, 30(2), 149-158.

Toro-Carvajal, L., Ortíz-Álvarez, H., Jiménez-García, F. y Agudelo-Calle J. (2012). Los sistemas cognitivos artificiales en la enseñanza de la matemática. Educación y Educadores, 15(2), 167-183.

Underhill, R. (1988). Mathematics learners' beliefs: A review. Focus on Learning Problems in Mathematics, 10(1), 55-69.

Vila, A. y Callejo, M. (2005). Matemáticas para aprender a pensar: El papel de las creencias en la resolución de problemas. Madrid: Narcea.

Villoro, L. (2006). Creer, saber, conocer. México: Siglo XXI.

Wells, C. y Wollack, J. (2003). An Instructor's Guide to Understanding Test Reliability. University of Wisconsin: Testing y Evaluation Services.

Yong, A.G. y Pearce, S. (2013). A beginner's guide to factor analysis: focusing on exploratory factor analysis. Tutorials in Quantitative Methods for Psychology 2013, 9(2),79-94.

Zambrano, G. (2008). Preguntas cognitivas y metacognitivas en el aprendizaje y la generación de estrategias de resolución de problemas matemáticos. Revista Inventum, 4, 25-50.

JoSE MANUEL DiEGo-MANTECón

Dirección: Universidad de Cantabria, Facultad de Ciencias Avda. de Los Castros 48. 39005 Santander, Cantabria (España)

Teléfono: (+34) 655457107 